\title{
MAIN CONTRACTOR - SUBCONTRACTOR RELATIONS IN THE FRAMEWORK OF CONTRACT THEORY
}

\author{
Iryna Nykyforchyn ${ }^{1}$, Nataliia Suduk ${ }^{2}$ \\ Vasyl Stefanyk Precarpathian National University, Ukraine
}

\begin{abstract}
The purpose of this paper is to study optimal relationships between customer, main contractor, and subcontractor. We make the following assumptions: main contractor and subcontractor have types that specify expenses and outcomes for all actions that can be performed. The higher type has fewer expenses for the same action, and optimal action increases with type. Contractors announce their types but can cheat, i.e., not reveal true types. The action and the respective remuneration are specified by the higher authority accordingly to the type declared. Incentives are paid so distorting real type is disadvantageous both for main contractor and subcontractor. We consider these assumptions to be compatible with economic practice. Methodology. We apply methods of contract theory, probability theory, and variation calculus, appropriately modifying apparatus of classic basic incentive problem. Variety of types of (sub-)contractors available is described via probability distributions. Then expected values of profits for participants are calculated as integral functionals. Maximization of these functional results in implicit equations for optimal incentive functions. Results. A method for optimization of activity of all participants is developed. Its requirements are traditional and non-restrictive; hence the expected area of applications is wide enough. Optimal actions and incentive functions are found. Formulae for the influence of expected main contractor and subcontractor's productivity and expenses on customers profit and payoffs are presented. Conclusions. It is shown that generally customer does not need to collect information about real subcontractor, relying on main contractor, but should take into account the actual situation in the respective branch. In the case when the customer is fully informed about this situation, contractor's expected profit does not depend on subcontractor's type (although it is only a mathematical expectation and a concrete result can vary).
\end{abstract}

Key words: contract theory, principal, agent, incentive compatibility, main contractor, subcontractor.

JEL Classification: D82, D86

\section{Introduction}

The organizational and economic mechanism for managing the region is a complex set of tools and processes affecting the social and market conditions of regional cooperation, which ensure the efficiency of the regional economy and improve the quality of life of the population (Blahun, Dmytryshyn, \& Leshuk, 2017). This mechanism carries out the following major large-scale processes: 1) direct management of the public sector of regional development; 2) coordination of various types of activities and processes of the socio-economic development of regions; 3) market stimulation of economic processes.

Stimulation is an external influence on an object, the motivating effect of which corresponds to its motives. Previously, economists paid less attention to how objects of economic activity respond to different incentives and, accordingly, how to create incentives for optimal behaviour. In the last third of the last century, this issue became the basis of economic research studying the economics of incentives, information economics and economy of contracts (Laffont \& Martimort, 2002). These areas are close enough and it is difficult to draw boundaries between them.

In the theory of contracts (Bolton \& Dewatripon, 2005), hierarchical structures with different amounts of power of the participants at different levels are considered, however, as a rule, incentive functions (principles of remuneration under contract fulfilment) are established by the highest governing centre, based on its interests. This agrees well with the realities of a large company but does not accurately describe the interaction of independent entities when the contractor independently optimizes the activities of the subcontractor, and the main customer does

\footnotetext{
Corresponding author:

${ }^{1}$ Department of Mathematics and Computer Science, Vasyl Stefanyk Precarpathian National University.

E-mail: ira.nyk@gmail.com

${ }^{2}$ Department of Economics, Vasyl Stefanyk Precarpathian National University.

E-mail:nsuduk@email.ua
} 
not always have full information about the final executors. Regional development requires the daily solution of production tasks that involve numerous business entities; in particular, contractors involve subcontractors, forming a branched contract contractual tree within each project. In today's realities, when management links are weakened, the main functions of regional governance are the decentralization of competences, the reduction of hierarchical levels of management with the consolidation of solved tasks and responsibilities, the clear separation of strategic and tactical tasks.

The purpose of our article is to analyse the relationship "customer - contractor - subcontractor", i.e., the "vertical links" in the hierarchy (cf. (Nykyforchyn, 2017) for analysis of "horizontal links"). The proposed model is rather schematic, however, allows us to draw some conclusions about the optimal behaviour of participants. We do not impose severe restrictions on functions that describe the costs and the effect of the participants, making only assumptions that are traditional for the theory of contracts.

\section{The main assumptions of the model}

Consider a model including a centre and two agents such that the centre is a principal for the first agent and the first agent is a principal for the second one. Each of the agents has his type (in fact, his productivity), which is known to him only and specified with a number $Q$ and $q$ respectively. An agent can inform his principal of another type $\hat{Q}$ or $\hat{q}$ (pretend to be of another type), cf. (Cabrales \& Charness, 2000).

According to the declared type $\hat{Q}$, the center requires the action $\Omega=\Omega(\hat{Q})$ from the first agent, and, after having it completed, pays $T(\hat{Q})$ (the function $T$ is previously announced to the first but not to the second agent, the function $\Omega$ is known to the principal only). Expenses of the first agent are reduced depending on the effect $h$ of the second agent's action and are equal to $C(Q, \Omega)-h$. The effect of the first agent's action is determined with a function $H(Q, \Omega)$.

The first agent, being the principal for the second one, requires the action $\theta=\theta_{Q}(\hat{q})$ respective to the type declared and pays the incentive $t_{Q}(\hat{q})$. Functions $\theta_{Q}$ and $t_{Q}$ depend on the parameter $Q$, hence we use equivalent denotations $\theta_{Q}(\hat{q})=\theta(q, Q)$ and $t_{Q}(\hat{q})=t(q, Q)$. Function $\theta_{Q}$ (but not the parameter $Q$ ) is previously announced to the second agent.

The second agent performs the required action, which expenses equal to $c(q, \theta)$, and reduces expenses of the first agent by $h=(q, \theta, \Omega)$.

Thus the profits are:

$$
\begin{aligned}
& R=H(Q, \Omega(\hat{Q})-T(\hat{Q})(\text { centre }) ; \\
& R_{1}=T(\hat{Q})-C(Q, \Omega(\hat{Q}))+h\left(q, \theta_{Q}(\hat{q}), \Omega(\hat{Q})\right)-t_{Q}(\hat{q}) \\
& \text { (first agent); } \\
& R_{2}=t_{Q}(\hat{q})-c\left(q, \theta_{Q}(\hat{q})\right) \text { (second agent). }
\end{aligned}
$$

We assume that the first agent's type is continuously distributed between $Q$ and $\bar{Q}$ with the density $f_{1}$ and the distribution function $F_{1}$. Similarly, let $f_{2}$ and $F_{2}$ be the density and the distribution function of the second agent's type between $\underline{q}$ and $\bar{q}$. These distributions are considered independent and known to all participants, as well as the functions $h, c, C$.

\section{Incentives for the second agent}

We also assume that the mentioned functions are such that that announcing incorrect type is disadvantageous. For the second agent, this results in the classic conditions

$$
\begin{aligned}
& \left.\frac{\partial R_{2}}{\partial \hat{q}}\right|_{q=\hat{q}}=t^{\prime}{ }_{Q}-c_{\theta}\left(q, \theta_{Q}(q)\right) \theta^{\prime}{ }_{Q}(q)=0 \cdot(\mathrm{IC} 1) \\
& \left.\frac{\partial^{2} R_{2}}{\partial \hat{q}^{2}}\right|_{q=\hat{q}}=t^{\prime \prime}{ }_{Q}-c_{\theta \theta}\left(q, \theta_{Q}(q)\right)\left(\theta^{\prime}{ }_{Q}(q)\right)^{2}- \\
& -c_{\theta}\left(q, \theta_{Q}(q)\right) \theta^{\prime \prime}{ }_{Q}(q)<0
\end{aligned}
$$

Taking the derivative of the first condition with respect to $\mathrm{q}$ and substituting into the second one, we obtain $\mathrm{t}^{\prime \prime}{ }_{\mathrm{Q}}-c_{\theta q}\left(q, \theta_{\mathrm{Q}}(q)\right) \theta_{\mathrm{Q}}^{\prime}(q)-c_{\theta \theta}\left(q, \theta_{\mathrm{Q}}(q)\right)^{2}-c_{\theta}\left(q, \theta_{\mathrm{Q}}(q)\right) \theta^{\prime \prime}{ }_{\mathrm{Q}}(q)=0$

Thus: $c_{\theta} q\left(q, \theta_{Q}(q)\right) \theta_{Q}^{\prime}(q)<0$.

Assuming Spence-Mirrlees condition $c_{\theta q}<0$, we obtain that in the pair of incentive compatibility conditions (IC1), (IC2) the second one can be replaced with the inequality $\theta_{Q}^{\prime}(q)>0$, i.e., the desired action of the second agent has to increase with its type. Having this satisfied, we can be sure that $\bar{q}=q$ and the derivative of the second agent's profit with respect to his type is equal to

$$
\begin{aligned}
& \frac{\partial R_{2}}{\partial \widehat{q}}=\underbrace{t^{\prime}{ }_{Q}-c_{\theta}\left(q, \theta_{Q}(q)\right) \theta^{\prime}{ }_{Q}(q)}_{=0}-c_{\theta}\left(q, \theta_{Q}(q)\right)= \\
& =-c_{\theta}\left(q, \theta_{Q}(q)\right) .
\end{aligned}
$$

Taking into account that expenses decrease with type, obtain $c_{q}<0$, hence $\frac{\partial R_{2}}{\partial \hat{q}}>0$, i.e., the profit $R_{2}>0$, which is called information rent, increases with his type.

It is natural also to require that the second agent's action is not unprofitable (individual rationality):

$$
R_{2}=t_{Q}(\hat{q})-c\left(q, \theta_{Q}(\hat{q})\right) \geq 0
$$

Clearly, it is sufficient for (IR) that the above inequality is valid only for the least value of $R_{2}$, i.e., for the lowest type $q$ of the second agent. Therefore the first agent prefers the least possible function $R_{2}$ such that $R_{2}=0$ for type $\underline{q}$, which implies that the information rent can be expressed via the calculated above derivative and the action function:

$$
R_{2}(q, Q)=-\int_{\underline{q}}^{q} c_{q}\left(\hat{q}, c_{q}(\hat{q})\right) \mathrm{d} \hat{q} .
$$

Thus, the optimal incentive function equals

$t_{\mathrm{Q}}(q)=c\left(q, \theta_{\mathrm{Q}}(q)\right)+R_{2}(q, Q)=c\left(q, \theta_{Q}(q)\right)-\int_{q}^{q} c_{q}\left(\hat{q}, c_{q}(\hat{q})\right) \mathrm{d} \hat{q}$.

Such reasoning is typical for the basic incentive problem (Grossman \& Hart, 1982; Grossman \& Hart, 1983). 


\section{Optimal action function of the second agent and incentives for the first agent}

Now consider the profit (the information rent) of the first agent in view of $\hat{q}=q$. For his type distortion to be disadvantageous, it is necessary that expected information rent has a maximum in $\widehat{Q}=Q$. Calculate this expectation:

$$
\begin{aligned}
& E R_{1}(\hat{Q})=\int_{\underline{q}}^{q} R_{2}(\hat{Q}, \hat{q}) f_{2}(q) \mathrm{d} q=-\int_{\underline{q}}^{q}(T(\hat{Q})- \\
& \left.-C(Q, \Omega(\hat{Q}))+h\left(q, \theta_{Q}(q), \Omega(\hat{Q})\right)-t_{Q}(q)\right) f_{2}(q) d q= \\
& =T(\hat{Q})-C(Q, \Omega(\hat{Q}))+\int_{\underline{q}}^{q}\left(h\left(q, \theta_{Q}(q), \Omega(\hat{Q})\right)-\right. \\
& \left.-c(q, \theta(q, Q))+\int_{\underline{q}}^{q} c q(\hat{q}, \theta(\hat{Q}, \hat{q})) f_{2}(q) \mathrm{d} \hat{q}\right)\left(f_{2}(q) \mathrm{d} q\right.
\end{aligned}
$$

Since all the summands of the latter expression but $h$ do not depend on $\hat{Q}$, we can by analogy to the above calculations show that similar incentive compatibility conditions have to be valid:

$$
\begin{aligned}
& \left.\quad \frac{\partial R_{1}}{\partial \hat{q}}\right|_{\hat{Q}=Q}=T^{\prime}(\hat{Q})-C_{\Omega}(Q, \Omega(Q)) \Omega^{\prime}(Q)+ \\
& +\int_{\underline{q}}^{\mathrm{q}} h_{\Omega}\left(q, \theta_{Q}(q), \Omega(Q)\right) \Omega^{\prime}(Q) f_{2}(q) \mathrm{dq}=0
\end{aligned}
$$

This is $(\mathrm{IC} 1+)$ condition.

$$
\begin{aligned}
& \left.\frac{\partial^{2} E R_{1}}{\partial \hat{Q}^{2}}\right|_{Q=\hat{Q}}=T^{\prime \prime}(\hat{Q})-C_{\Omega \Omega}(Q, \Omega(Q))\left(\Omega^{\prime}(Q)\right)^{2}+ \\
& +C_{\Omega}(Q, \Omega(Q)) \Omega^{\prime \prime}(Q) \int_{\underline{q}}^{q}\left(h_{\Omega \Omega}\left(q, \theta_{Q}(q), \Omega(Q)\right)\right. \\
& \Omega^{\prime}(Q)+h_{\Omega}\left(q, \theta_{Q}(q), \Omega(Q) \Omega^{\prime \prime}(Q)\right) f_{2}(q) \mathrm{dq}<0
\end{aligned}
$$

We again take the derivative of the first condition with respect to $\mathrm{Q}$ and substitute the result into the second one, hence

\section{$C_{\Omega \Omega}(Q, \Omega(Q)) \Omega^{\prime}(Q)<0$}

and, assuming Spence-Mirrlees condition $C_{\Omega Q}<0$ also to be valid, obtain an equivalent inequality $\Omega^{\prime}(Q)>0$, which means that the desired action of the first agent increases with his type.

Note that the "utility" $h$ of the second agent for the first one depends on the both agents' actions and the second (but not the first) agent's type. Due to such "splitting" the function $h$ does not take part in the simplified form of the condition (IC2+).

Assuming incentive compatibility conditions to hold, consider the possibility of maximization of $E R_{1}(Q)$ for $\hat{Q}=Q$, and, respectively, $\Omega(Q)$ already fixed.

Change the order of integration:

$$
\begin{aligned}
& \int_{\underline{q}}^{\bar{q}}(h(q, \theta(q, Q), \Omega(Q)))-c(q, \theta(q, Q))+ \\
& \left.\left.+\int_{\underline{q}}^{\mathrm{q}} c_{\mathrm{q}}(\hat{q}, \theta(\hat{q}, Q)) \mathrm{d} \hat{q}\right) f_{2}(q)\right)\left(f_{2}(q) \mathrm{dq}\right. \\
& =\int_{\underline{q}}^{q}(h(q, \theta(q, Q), \Omega(Q)))-c(q, \theta(q, Q)) f_{2}(q) \mathrm{dq}+ \\
& \left.\int_{\underline{q}}^{\bar{q}}\left(\int_{q}^{\bar{q}} c_{\mathrm{q}}(q, \theta(q, Q)) \mathrm{d} \hat{q}\right) f_{2}(\hat{q})\right)\left(f_{2}(q) \mathrm{d} \hat{q}\right) \mathrm{dq}= \\
& \int_{\underline{q}}^{\bar{q}}(h(q, \theta(q, Q), \Omega(Q)))-c(q, \theta(q, Q)) f_{2}(q) \mathrm{dq}+ \\
& +\int_{\underline{q}}^{\bar{q}} \int_{q}^{\bar{q}} c_{\mathrm{q}}\left(q, \theta_{Q}(q, Q)\right)\left(1-F_{2}(q)\right) d q
\end{aligned}
$$

To maximize this functional while $\theta_{Q}$ runs through all non-decreasing continuous functions, we equate to zero the derivative of the integrand with respect to action, hence

$$
\begin{aligned}
& (\mathrm{h}(\mathrm{q}, \theta(\mathrm{q}, \mathrm{Q}), \Omega(\mathrm{Q})))-\mathrm{c}(\mathrm{q}, \theta(\mathrm{q}, \mathrm{Q})) \mathrm{f}_{2}(\mathrm{q})+\mathrm{c}_{\mathrm{q} \theta}\left(\mathrm{q}, \theta_{\mathrm{q}}(\mathrm{q})\right. \\
& \left(1-\mathrm{F}_{2}(\mathrm{q})\right)=0 .
\end{aligned}
$$

Solving this equation with respect to $\Omega$ for all $q$ with Q being a parameter, we obtain a function $\theta_{\Omega}(Q)$, which is the optimal action that the first agent will require from the second one. Observe that the latter equation depends not directly on $Q$, but on the action $\Omega=\Omega(Q)$ that the second agent should perform, therefore, the optimal for the first agent action of the second agent is a solution $\theta_{\Omega}(Q)=\theta^{\Omega}$ of the equation

$$
\begin{aligned}
& \left(h_{\theta}\left(q, \theta^{\Omega}(q), \Omega\right)-c_{\theta}\left(q, \theta^{\Omega}(q)\right)\right) f_{2}(q)+c_{q \theta}\left(q, \theta^{\Omega}(q)\right) \\
& \left(1-F_{2}(q)\right)=0
\end{aligned}
$$

Recall that due to (IC2) $\theta^{\Omega}$ should satisfy the requirement $\frac{\partial \theta^{\Omega}}{\partial q}>0$ for all $\Omega$.

Calculate the derivative

$$
\begin{aligned}
& \frac{\partial E R_{1}}{\partial \hat{q}}=\underbrace{T^{\prime}(\hat{Q})-C_{\Omega}(Q, \Omega(Q)) \Omega^{\prime}(Q)+\int_{\underline{q}}^{\bar{q}} h_{\Omega}\left(q, \theta_{Q}(q), \Omega(Q)\right) \Omega^{\prime}(Q)}_{=0}-C_{Q}(Q, \Omega(Q))+ \\
& \int_{\underline{q}}^{\bar{q}} \frac{\partial \theta(q, Q)}{\partial \hat{q}} \underbrace{\left(h_{\theta}\left(q, \theta^{\Omega}(q), \Omega\right)-c_{\theta}\left(q, \theta^{\Omega}(q)\right)\right) f_{2}(q)+c_{q \theta}\left(q, \theta^{\Omega}(q)\right)\left(1-F_{2}(q)\right)}_{=0} d q=-C_{Q}(Q, \Omega(Q))
\end{aligned}
$$

Again positivity is obtained, hence the mathematical expectation of the first agent's information rent also increases with his type independently of the form of "utility function" of the second agent for the first one. Only optimality of stimulation of the first agent by the centre and of the second agent by the first one is sufficient.
The (IR+) incentive compatibility condition $E R_{1} \geq 0$ for the first agent can be verified for his lowest type $Q$ only, therefore, we can put $E R_{1}(Q)=0$ and, taking into account the above-calculated derivative, obtain

$E R_{1}(Q)=-C_{Q}(Q, \Omega(Q))$,

hence the incentive function is: 


$$
\begin{aligned}
& T(Q)=C\left(Q, \Omega(Q)-\int_{\underline{Q}}^{Q} \mathrm{C}_{Q}((\hat{q}), \Omega(\hat{Q}) \mathrm{d} \hat{Q}\right. \\
& -\int_{\underline{q}}^{\bar{q}}\left(\left(h\left(q, \theta^{\Omega(Q)}(q), \Omega(Q)-c\left(q, \theta^{\Omega(Q)}(q)\right)\right) f_{2}(q)\right.\right. \\
& \left.+c_{q}\left(q, \theta^{\Omega(Q)}(q)\right)\left(1-F_{2}(q)\right)\right) d q
\end{aligned}
$$

and is determined with the selected increasing action function $Q^{\Omega}$.

\section{Optimization of the centre profit}

Which is left is to find a function $\Omega(Q)$ that maximizes the expected centre profit.

$$
\begin{aligned}
& E R=E(H(Q, \Omega(Q))-T(Q)) \\
& =\int_{\underline{Q}}^{\bar{Q}}\left(H(Q, \Omega(Q))-C(Q, \Omega(Q))+\int_{\underline{Q}}^{Q} C_{Q}(\widetilde{Q}, \Omega(\widetilde{Q})) d \widetilde{Q}\right. \\
& +\int_{\underline{q}}^{\bar{q}}\left(\left(h\left(q, \theta^{\Omega(Q)}(q), \Omega(Q)-c\left(q, \theta^{\Omega(Q)}(q)\right)\right) f_{2}(q)\right.\right. \\
& \left.\left.+c_{q}\left(q, \theta^{\Omega(Q)}(q)\right)\left(1-F_{2}(q)\right)\right) d q\right) f_{1}(Q) d q \\
& =\int_{\underline{Q}}^{\bar{Q}}\left(f_{1}(Q) H(Q, \Omega(Q))-C(Q, \Omega(Q))\right. \\
& +\int_{\underline{q}}^{\bar{q}}\left(\left(h\left(q, \theta^{\Omega(Q)}(q), \Omega(Q)-h\left(q, \theta^{\Omega(Q)}(q)\right)\right) f_{2}(q)\right.\right. \\
& \left.\left.+c_{q}\left(q, \theta^{\Omega(Q)}(q)\right)\left(1-F_{2}(q)\right)\right) d q\right) f_{1}(Q) d q+ \\
& \left.+C_{Q}(Q, \Omega(Q))\left(1-F_{1}(Q)\right)\right) d Q
\end{aligned}
$$

Taking derivative with respect to $\Omega$, obtain an equation for the action function:

$$
\begin{aligned}
& f_{1}(Q)\left(H_{\Omega}(Q, \Omega(Q))-C_{Q}(Q, \Omega(Q))+\int_{\underline{q}}^{\bar{q}} \frac{\partial \theta}{\partial \Omega}(q, \Omega(Q))\right) \\
& \left(\left(h_{\theta}\left(q, \theta^{\Omega(Q)}, \Omega(Q)-c_{q}\left(q, \theta^{\Omega(Q)}(q)\right)\right) f_{2}(q)+\right.\right. \\
& \left.+c_{q \theta}\left(q, \theta^{\Omega(Q)}(q)\right)\left(1-F_{2}(q)\right)\right) d q+\int_{\underline{q}}^{\bar{q}} h_{\Omega}\left(q, \theta^{\Omega(Q)}(Q)\right. \\
& f_{2}(q) d q+C_{Q \Omega}(Q, \Omega(Q))\left(1-F_{1}(q)\right)=0
\end{aligned}
$$

In view of the equation $\theta^{\Omega}$, we see that the first integral is zero, hence the equation becomes of the form similar to the form of the equation for the second agent's action but with a summand depending on the function $h$ and $\theta$ :

$$
\begin{aligned}
& f_{1}(Q)\left(H_{\Omega}(Q, \Omega(Q))-C_{Q}(Q, \Omega(Q))+\int_{q}^{\bar{q}} h_{\Omega}\left(q, \theta^{\Omega(Q)}\right.\right. \\
& (Q) f_{2}(q) d q+C_{Q \Omega}(Q, \Omega(Q))\left(1-F_{1}(q)\right)=0
\end{aligned}
$$

The functional of expected centre profit can have a maximum in its solution if it satisfies the condition
$\Omega^{\prime \prime}(Q)>0$. The latter equation implies that the optimal action of the first agent that is selected by the centre depends on the expected productivity of the second agent, with the exception of the degenerate case when the utility of the second agent is the same for all actions of the first agent. On the other hand, this productivity affects the expected centre profit according to the above formula.

\section{Conclusions}

Under rather general assumptions, we have obtained methods for optimization of activity of all participants in the presented model. The derived formulae allow performing computational experiments for specific types of functions describing expenses and productivity of main contractor and subcontractor. At the same time, certain conclusions can be drawn even without such concretization.

First, if control is "incentive compatible", then main contractor's expenses on payments to a subcontractor are on the average compensated by the customer; hence the expected profit of the main contractor depends on his own properties only, i.e., on expenses and utility for the customer.

Second, optimal action required from the contractor accordingly to its type, as well as expected centre profit, generally depends on expected subcontractor properties.

This means that there is no need for the customer to collect information about real subcontractor. The customer can rely on the main contractor in the optimization of subcontractor activity but should take into account the actual situation in the respective branch (in the form of the probability distribution of the parameter that specified expenses and productivity). This conclusion correlates with the main result of (Melumad, Mookherjee, \& Reichelstein, 1995), who also suggested contracting delegation.

Describe explicitly assumptions of the model necessary for the conclusions drawn to be true. Customer and contractor define incentive function so that true types of agents are revealed. Contractor accepts contract before the true type of a subcontractor is known and incentive function for subcontractor is announced after incentive function for the contractor (so the customer cannot guess contractor's real type). Expenses functions and effect functions (but not the types that are parameters of these functions) are known to all participants.

We do not consider possibilities of participants' coalitions.

Further research will focus on computational experiments and on verifying that the Revelation Principle does not prevent us from finding more effective strategies for the participants. 


\section{References:}

Blahun I., Dmytryshyn L. \& Leshuk H. (2017). Simulative model for evaluation of investment processes in the regions of Ukraine. Investment Management and Financial Innovations, 14(3), 322-329.

Bolton P. \& Dewatripon M. (2005). Contract Theory - Cambridge, Mass \& London, England: MIT Press.

Cabrales A. \& Charness G. (2000). Optimal Contracts, Adverse Selection and Social Preferences: An Experiment. Economics Working Papers 478, Department of Economics and Business, Universitat Pompeu Fabra.

Grossman S. \& Hart O. (1982). Implicit Contracts Under Asymmetric Information. Quarterly Journal of Economics, 1, 110-124.

Grossman S. \& Hart O. (1983). An Analysis of the Principal-Agent Problem. Econometrica, 51(1), 7-45.

Laffont J.-J. \& Martimort D. (2002). The Theory of Incentives. The Principal - Agent Model - Princeton \& Oxford: Princeton University Press.

Melumad D.N., Mookherjee D. \& Reichelstein S. (1995). Hierarchical Decentralization of Incentive Contracts. The Rand Journal of Economics, 26(4), 654-672.

Nykyforchyn I.V. (2017). Simulation of Active Systems with Dependent Incentives of Agents, Herold of Volyn Institute for Economics and Management, 19, 178-184. (in Ukrainian) 